\title{
Modeling and Performance Analysis of Route-Over and Mesh-Under Routing Schemes in 6LoWPAN under Error-Prone Channel Condition
}

\author{
Tsung-Han Lee, ${ }^{1}$ Hung-Chi Chu, ${ }^{2}$ Lin-Huang Chang, ${ }^{1}$ \\ Hung-Shiou Chiang, ${ }^{1}$ and Yen-Wen Lin ${ }^{1}$ \\ ${ }^{1}$ Department of Computer Science, National Taichung University of Education, No. 140, Minsheng Road, West District, \\ Taichung 40306, Taiwan \\ ${ }^{2}$ Department of Information and Communication Engineering, Chaoyang University of Technology, No. 168, Ji-Fong East Road, \\ Wu-Fong District, Taichung 41349, Taiwan \\ Correspondence should be addressed to Tsung-Han Lee; thlee@mail.ntcu.edu.tw
}

Received 21 June 2013; Accepted 20 August 2013

Academic Editor: Chih-Hao Lin

Copyright (c) 2013 Tsung-Han Lee et al. This is an open access article distributed under the Creative Commons Attribution License, which permits unrestricted use, distribution, and reproduction in any medium, provided the original work is properly cited.

6LoWPAN technology has attracted extensive attention recently. It is because 6LoWPAN is one of Internet of Things standard and it adapts to IPv6 protocol stack over low-rate wireless personal area network, such as IEEE 802.15.4. One view is that IP architecture is not suitable for low-rate wireless personal area network. It is a challenge to implement the IPv6 protocol stack into IEEE 802.15.4 devices due to that the size of IPv6 packet is much larger than the maximum packet size of IEEE 802.15.4 in data link layer. In order to solve this problem, 6LoWPAN provides header compression to reduce the transmission overhead for IP packets. In addition, two selected routing schemes, mesh-under and route-over routing schemes, are also proposed in 6LoWPAN to forward IP fragmentations under IEEE 802.15.4 radio link. The distinction is based on which layer of the 6LoWPAN protocol stack is in charge of routing decisions. In route-over routing scheme, the routing distinction is taken at the network layer and, in mesh-under, is taken by the adaptation layer. Thus, the goal of this research is to understand the performance of two routing schemes in 6LoWPAN under error-prone channel condition.

\section{Introduction}

6LoWPAN $[1,2]$ is an IETF standardized IPv6 adaptation layer that allows IP over low-power, lossy networks. Extending IP to LoWPANs (low-power, wireless personal area networks) faces different challenges than traditional network. The microcontrollers typically embedded with LoWPAN radios have limited memory and compute power. Thus, the technique for transmitting IPv6 packets over Low-power Wireless Personal Area Networks is called 6LoWPAN. However, 6LoWPAN is difficult to implement because the size of IPv6 packet is much larger than the packet size of IEEE 802.15.4 data link layer. In order to make it possible, the IETF 6LoWPAN working group introduces the adaptation layer between network and data link layers. It provides header compression to reduce transmission overhead, fragmentation, and reassembly of IPv6 packet. It can also be involved in routing decisions, and the routing scheme in 6LoWPAN can be divided into two categories. In the mesh-under method, the routing decision is taken in adaptation layer. On the other hand, the route-over method makes the routing decision in network layer.

Mesh-under and route-over routing schemes can be considered as end-to-end and hop-by-hop transmission, respectively. Hop-by-hop fragmentation and reassembly generate more delay but achieve better fragment arrival ratio. Whereas end-to-end scheme has less latency, but fragment loss has high probability. Therefore, the goal of this research is to understand the performance of two routing schemes in 6LoWPAN under error-prone channel condition. 
In 6LoWPAN protocol stack, the last two layers are based on IEEE 802.15.4 physical and data-link layers. A two-dimensional discrete-time Markov chain for unslotted CSMA/CA mechanism in 6LoWPAN is used to analyze the performance in unsaturated 6LoWPAN for rout-over and mesh-under routing schemes. Based on this Markov chain, the packet successful transmission probability for route-over and mesh-under routing schemes is evaluated under different numbers of competing nodes and wireless channel condition. To the best of our knowledge, this study is not yet reported in the literature. Finally, we attempt to compare route-over against mesh-under routing schemes in 6LowPAN in terms of goodput for IP end-to-end communication.

The rest of the paper is organized as follows. Next section describes related research works. Section 3 introduces the 6LoWPAN unslotted CSMA/CA mechanism. Section 4 describes the mesh-under and the route-over routing schemes in 6LoWPAN. Section 5 describes the proposed twodimensional discrete-time Markov chain to model 6LowPAN unslotted CSMA/CA mechanism and analyzes the goodput for 6LowPAN routing schemes under error-prone channel condition. Section 6 shows the analysis results for probability of IP packet successful transmission in both route-over and mesh-under routing schemes. Finally, Section 7 presents our conclusions.

\section{Related Work}

In the case of the WLAN, Bianchi [3] presented an analytical model to compute the saturation throughput performance evaluation of both RTC/CTS access mechanisms.

In WSN, [4] presented a similar analytical model to predict energy consumption as well as the throughput of saturated and unsaturated 802.15.4 networks, based on which some design guidelines can be derived. In order to address system goodput and energy efficiency enhancement, [5] studies packet size optimization for IEEE 802.15.4 networks. Taking into account the CSMA-CA contention, protocol overhead, and channel condition, analytical models are proposed to calculate the goodput and the energy consumption. In [6], the authors try to analyze the complete CSMA/CA in IEEE 802.15.4. First, to analyse the performance of the slotted CSMA/CA of IEEE 802.15 .4 by integrating the discrete-time Markov chain models of the node states and the channel states and then extend the models by adopting a modification to the CAP. The extended models could be used to analyze the performance of the unslotted and slotted CSMA/CA strategy. In nonbeacon-enabled mode [7], build a process chain to model unslotted CSMA/CA mechanism. However, the backoff procedure is not only a Markov chain but also the backoff time counter is an accumulation which value depends on how many times the node has tried to access the channel without success. According to the proposed process chain and mathematical model, the distribution of traffic changes has been estimated when different loads are offered to the network. Moreover, the proposed model can evaluate the proper packet size to improve the success probability.
In [8], an analytical comparison between route-over and mesh-under schemes based on 6LoWPAN experimental research which tries to analyze the fragment arrival probability, the total number of transmissions and the total delay from source and destination. However, the authors analyze the arrival probability for single fragment in multihop environment. Furthermore, they assume that the fragment arrival probability for one hop distance is fixed, which means that the number of competing nodes in each hop has not been concerned. In our research, the probability of successful transmission for each fragment is different depending on the contention window in MAC layer for IEEE 802.15.4. And we analyze the complete IP packet arrival probability including all IP fragments for route-over and mesh-under.

\section{Overview of CSMA/CA Mechanism in 6LowPAN}

6LoWPAN protocol stack adopts IEEE 802.15.4 standard $\mathrm{PHY}$ and MAC layers which are specified in $[1,2]$. Figure 1 [9] illustrates the steps of unslotted CSMA/CA algorithm of 6LoWPAN. NB is the number of times the CSMA/CA algorithm was required to back off while attempting the current transmission (the value will be initialized to 0 ). In the first-time backoff, the range of random contention window $(\mathrm{CW})$ is from $\left[0,2^{\text {macMinBE }}-1\right]$. Here macMinBE is the minimum value of the backoff exponent (BE) that has the default value which is 3 . When backoff counter reaches 0 , the node performs channel sensing immediately.

The random backoff mechanism is used to decrease the probability of collisionsand ensure that the channel is clear for a node to access it. The channel clear assessment in unslotted CSMA/CA is one backoff period (in slotted CSMA/CA, which performs two channel clear assessments before transmission). If the channel is detected to be busy, $\mathrm{BE}$ is increased by 1 , and the new backoff stage begins before channel sensing. This process is repeated until BE equals upperbounded macMaxBE (maximum value of $\mathrm{BE}$, the default is 5), and then the BE is frozen at macMaxBE. When the number of backoff stage is equal to macMaxCSMABackoffs (the default value is 4 ), the node access channel is failure.

\section{Routing Scheme in 6LoWPAN}

To enable the transmission of large IPv6 packets over size constrained link layer payload size (102 bytes of payload) in IEEE 802.15.4, the 6LoWPAN adaptation layer provides IP packet fragmentation mechanism [1]. All fragments are transmitted into multiple link-layer frames for reassembling them at the other end under the mesh-under or route-over routing scheme in 6LoWAN.

As mentioned in the previous section, 6LoWPAN divides routing schemes into mesh-under and route-over [2, 8] schemes. The distinction is based on which layer of the 6LoWPAN protocol stack is in charge of routing decisions; in route over they are taken at the network layer, and in mesh under at the adaptation layer. Figure 2 shows routing decision 


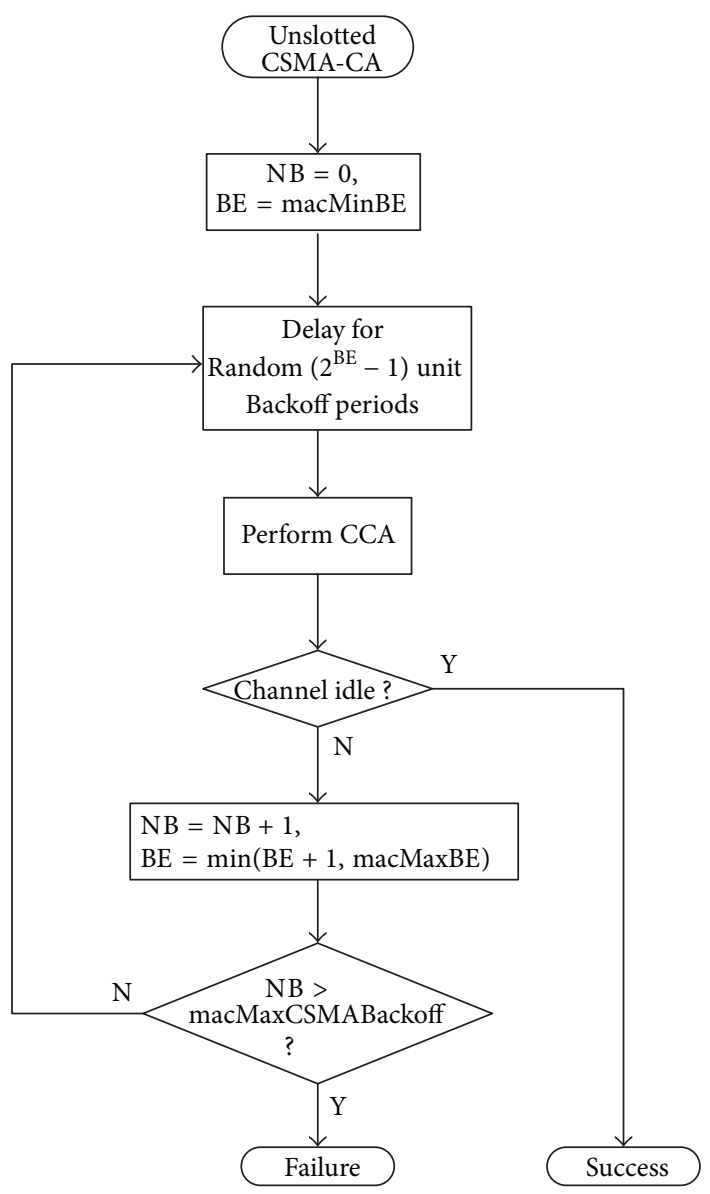

FIGURE 1: 6LoWPAN unslotted CSMA/CA algorithm [9].

layer for both mesh-under and route-over routing schemes [1].

4.1. Mesh-Under Routing Scheme. In the mesh-under routing scheme, the routing functions are placed at the link layer based on IEEE 802.15.4 frame structure and the 6LoWPAN header $[2,8]$. All fragments will be sent to the next hop by mesh routing and finally reach to the destination. Different fragments of one IP packet might reach the destination via different route-paths. If all fragments are received at the destination successfully, the destination's adaptation layer reassembles all fragments into an IP packet. The adaptation layer of destination node starts reconstruction process. However, any fragment is missing in forwarding process; all fragments of this IP packet are retransmitted from the source to the destination.

4.2. Route-Over Routing Scheme. In route-over scheme, each sensor node inside the route path acts as an IP router. The IP packet is forwarded hop by hop from the source node to the destination node $[2,8]$. The IP packet's payload is encapsulated with IPv6 header. After that, IP packet is fragmented by the adaptation layer and all IP fragments will be sent to the next hop based on routing table. The next hop has to reassemble them in order to reconstruct the original
IP packet in adaptation layer when all fragments are received successfully. The reconstruction process starts only when the last fragment arrives. Once reconstructed, the IP packet will be sent to the network layer. Finally, the IP packet will be fragmented again and these fragments will be delivered to the nexthop. However, the retransmission executes only in onehop distance if there is any fragment lost in this forwarding process.

\section{Numerical Analytical Model for 6LoWPAN Routing Schemes in Error-Prone Channel Condition}

In this section, we propose mathematical models to analyze the IP packet successful transmission probability for routeover and mesh-under, respectively. In addition, we present the goodput analysis to compare the performance of these two routing schemes under error-prone channel condition.

5.1. Markov Chain Model for Unslotted CSMA/CA Mechanism in Error-Prone Channel Condition. In Figure 3, a twodimensional discrete-time Markov chain model has been used to analyze the unslotted CSMA/CA mechanism in 6LoWPAN under error-prone channel condition. Define the 


\begin{tabular}{|c|}
\hline Application layer \\
\hline Transport layer \\
\hline Network layer \\
\hline Adaptation layer \\
\hline IEEE 802.15.4 \\
MAC layer \\
\hline $\begin{array}{c}\text { IEEE 802.15.4 } \\
\text { PHY layer }\end{array}$ \\
\hline $\begin{array}{c}\text { Mesh-under } \\
\text { routing scheme } \\
\text { Routing decision }\end{array}$
\end{tabular}

\begin{tabular}{|c|}
\hline Application layer \\
\hline Transport layer \\
\hline Network layer \\
\hline Adaptation layer \\
\hline $\begin{array}{c}\text { IEEE 802.15.4 } \\
\text { MAC layer }\end{array}$ \\
\hline $\begin{array}{c}\text { IEEE 802.15.4 } \\
\text { PHY layer }\end{array}$ \\
\hline $\begin{array}{c}\text { Route-over } \\
\text { routing scheme }\end{array}$ \\
\hline
\end{tabular}

FIGURE 2: Routing decision layer for both mesh-under and route-over routing schemes in 6LoWPAN [1].

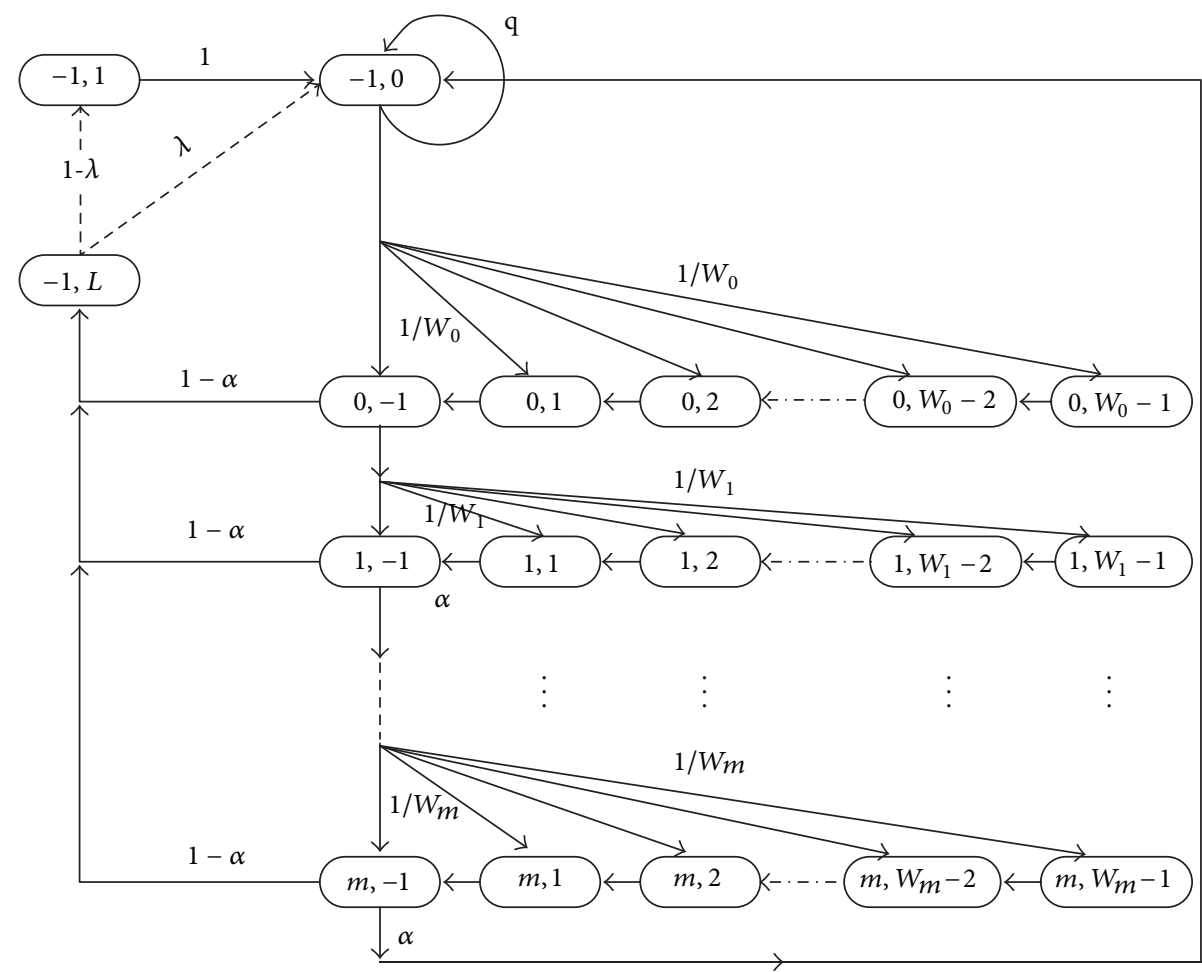

FIgURE 3: The Discrete-time Markov chain model for unslotted CSMA/CA mechanism in 6LoWPAN under error-prone channel condition.

state as $\{n b(t), b c(t)\} ; n b(t), b c(t)$ as the stochastic process representing the number of backoff times and the backoff counter at time slot $t$, respectively. When $n b(t) \in[0, m]$, $m$ is the maximum number of backoff stage which is equal to macMaxCSMABackoffs (the default value is 4 ); $b c(t) \epsilon$ $\left[0, W_{i}-1\right]$. Note that when $b c(t)=0$, the node enters CCA state immediately. Thus, $b c(t)=0$ is replaced by $b c(t)=-1$ which represents the successful CCA attempt. In addition, $\{0,-1\} \sim\{m,-1\}$ represents the successful CCA state for backoff stage from 0 to the maximum backoff number $m$.
According to the protocol, the duration of the backoff counter is

$$
W_{i}=2^{\operatorname{macMinBE}} 2^{\min \{\operatorname{macMaxBE}-\operatorname{macMinBE}, i\}}, \quad i \in[0, m]
$$

$\alpha$ is the probability that the channel is busy at the CCA detection. When $(t)=-1$, it represents the successful transmission attempt. The range of $n b(t)$ is from $\{-1, L\}$ to $\{-1,0\}$, where $L$ is the number of time slots when a packet is under the transmission duration. Hence, the length of a 
transmission period must equal the length of packet. We propose an interference model in the transmission process based on this Markov chain, it occurs when the transmission process. We let $\lambda$ be defined as frame error rate (FER). Bit error rate (BER) is used to indicate the wireless channel condition. Thus, the given certain BER value, $\lambda$, can be calculated by

$$
\begin{gathered}
\lambda=1-(1-\mathrm{BER})^{l} \\
l=L \cdot T_{\text {Slot }} \cdot R
\end{gathered}
$$

where $T_{\text {Slot }}$ is a aUnit Backoff Period and $R$ is a physical layer bit rate. Equation (2) is shown that the different channel conditions and the length of packet transmission will impact the FER. While the value of $\lambda$ is too high, the packet transmission error, a node, will back to the idle state $\{-1,0\}$. And $q$ is defined as the probability that the user is still in the idle state in the next time slot.

Let the stationary probabilities of the Markov chain be $b_{n, b}=P\{(n b(t), b c(t))=(n, b)\}$. Note that backoff counter reaches 0 and the node enters CCA state immediately. Hence, $b_{n, 0}$ has a same value as $b_{n,-1}$. We obtain that

$$
\begin{gathered}
b_{n,-1}=\alpha^{n} b_{0,-1}, \quad n \in[1, m], \\
b_{-1, L}=\left(1-\alpha^{m+1}\right) b_{0,-1}, \\
b_{-1,0}=\frac{b_{0,-1}}{1-q} .
\end{gathered}
$$

The sum of probabilities of all the states should be equal to 1 , and we have

$$
\begin{aligned}
& b_{-1,0}+\sum_{l=1}^{L} b_{-1, l}+\sum_{n=1}^{m} b_{n,-1} \\
& +b_{0,-1}+\sum_{n=0}^{m} \sum_{b=1}^{W_{n}-1} b_{n, b}=1,
\end{aligned}
$$

where $\tau$ is the probability that a node attempts carrier sensing; we get

$$
\tau=\sum_{n=0}^{m} b_{n,-1}
$$

Assume that the system has $N$ nodes. From [4], the probability that the channel is busy in CCA is

$$
\begin{aligned}
\alpha & =\left[1-\frac{1}{1\left(1+1 /\left(1-(1-\tau)^{N}\right)\right)}\right]\left(1-(1-\tau)^{N-1}\right) \\
& =\frac{1-(1-\tau)^{N-1}}{2-(1-\tau)^{N}} .
\end{aligned}
$$

According to the proposed Markov chain model, we can get the probability to enter transmission stage which is

$$
P_{\mathrm{tr}}=\tau \cdot(1-\alpha) .
$$

While a node access channel is successful, it will transmit data, and the transmission task is completed in data link layer. But this transmission cannot ensure that the packet arrival to receiver is correctly. It is possible occurring interference in air propagation. Hence, a successful transmission will not have any FER from sender to receiver. We can get

$$
P_{\text {suc }}=P_{\text {tr }} \cdot(1-\lambda) \text {. }
$$

5.2. IP Packet Successful Transmission Probability for RouteOver and Mesh-Under. Consider

$$
P_{\text {suc }}^{\mathrm{mu}}=\left\{\left[\sum_{i=0}^{k}\left(P_{\text {suc }}\right)\left(1-P_{\text {suc }}\right)^{i}\right]^{f}\right\}^{h} \text {. }
$$

Let $k$ represent macMaxFrameRetries which has default value which is 3 , and $f$ is the number of fragments with hop counts $h$. In mesh-under scheme, all fragments must aggregate at destination. Each fragment is sent from source to the destination in $h$ hops. Thus, the IP packet successful transmission probability decreases gradually after $f$ number of fragments through $h$ hops transmission route-path:

$$
P_{\text {suc }}^{\text {ro }}=\left[\sum_{i=0}^{k}\left(P_{\text {suc }}\right)\left(1-P_{\text {suc }}\right)^{i}\right]^{f} \text {. }
$$

Equation (11) is the IP packet successful probability of 6LoWPAN route-over routing scheme. The major feature of route-over is hop-by hop fragmentation and reassembly. In each hop, all fragments will recover to a completed IPv6 packet. Moreover, in the modeling assumption, each hop contents ideal channel condition. Consequently, we can consider route-over scheme as hop-by-hop forwarding from source to destination. Although this scheme consumes more energy and delay time, but it brings the robust packet transmission rate.

5.3. Goodput Analysis for 6LoWPAN Routing Schemes. To evaluate the goodput, we consider that a cycle of transmission includes idle, contention, and transmission states. These states define as the duration, and each one is normalized which contains probability. The equations are shown as follows.

$$
E \text { [idle }]=\left(1-P_{\text {tr }}\right)^{N} \cdot \sigma,
$$

where $\sigma$ is defined as the duration of an empty slot time and $N$ is number of competing nodes. Equation (12) is the expectation of node's idle duration. If any competing node generates frame to transmit, it will transit to contention state. The expectation of a node in contention state is

$$
\begin{aligned}
E[\text { wait }]= & P_{\mathrm{tr}} \cdot\left(7 \times T_{\text {Slot }}+T_{\mathrm{CCA}}\right) \\
& +P_{\mathrm{tr}} \cdot\left(1-P_{\mathrm{tr}}\right) \cdot\left(23 \times T_{\text {Slot }}+T_{\mathrm{CCA}}\right) \\
& +P_{\mathrm{tr}} \cdot\left(1-P_{\mathrm{tr}}\right)^{2} \cdot\left(55 \times T_{\text {Slot }}+T_{\mathrm{CCA}}\right) \\
& +P_{\mathrm{tr}} \cdot\left(1-P_{\mathrm{tr}}\right)^{3} \cdot\left(87 \times T_{\text {Slot }}+T_{\mathrm{CCA}}\right) \\
& +P_{\mathrm{tr}} \cdot\left(1-P_{\mathrm{tr}}\right)^{4} \cdot\left(119 \times T_{\text {Slot }}+T_{\mathrm{CCA}}\right) .
\end{aligned}
$$




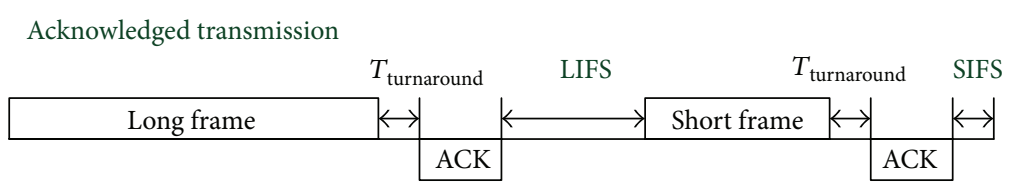

Unacknowledged transmission

LIFS SIFS

Long frame $\quad \longrightarrow$

FIGURE 4: The procedure of data transmission in 6LoWPAN using the acknowledged and unacknowledged transmission.

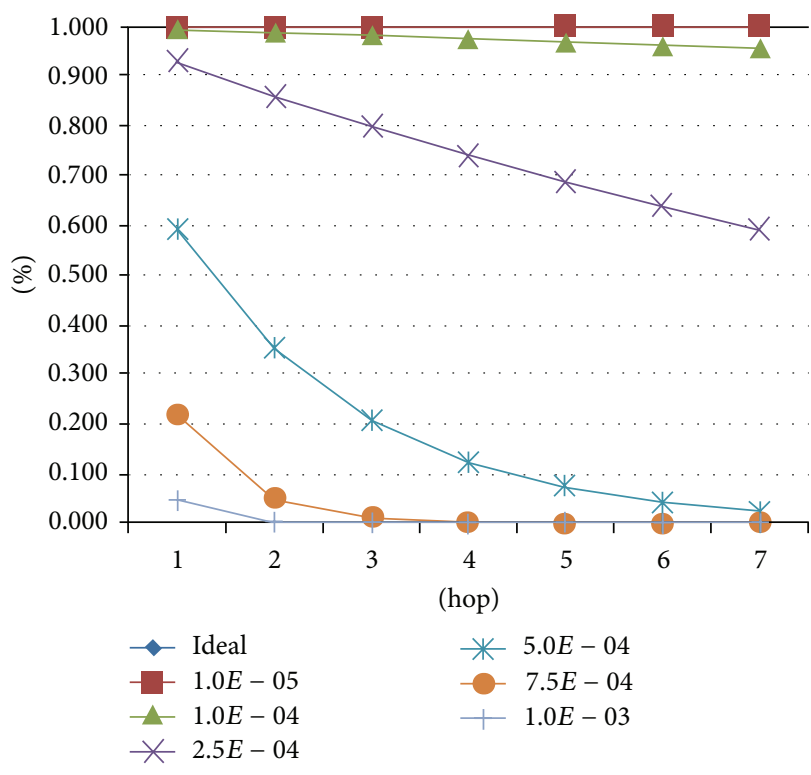

(a) Mesh-under

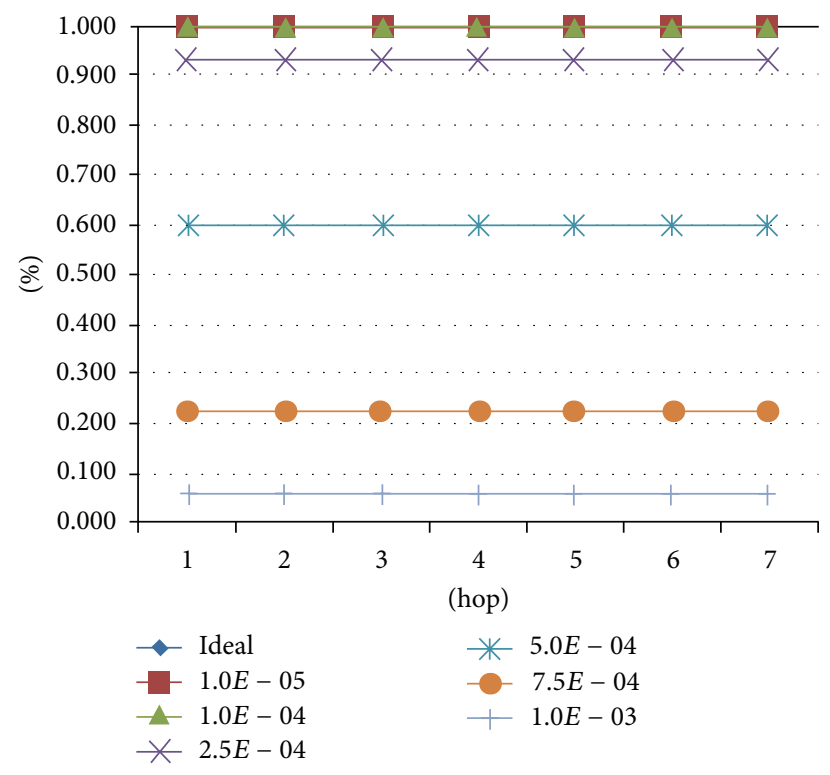

(b) Route-over

FIGURE 5: The successful IP packet transmission probability with 3 competing nodes for mesh-under and route-over routing schemes in 6LowPAN under error-prone channel condition.

In (13), a timeslot and CCA durations are $320 \mu \mathrm{s}$ and $128 \mu \mathrm{s}$, respectively. In the first backoff stage, the maximum number of slots for backoff counter is 8 (from 0 to 7 ). If access channel is failure in first stage, it will enter to next stage and backoff again. Since the main difference between route-over and mesh-under schemes is hop-by-hop fragmentation and reassembly, it means that the transmission delay in route-over scheme is higher than mesh-under scheme. Thus, we define $\delta$ is the delay time for the IP packet assembly and reassembly for route-over scheme. The expectation of transmission state for these two schemes is different. Moreover, we also consider the transmission can be with and without retransmission, respectively. From [9], the duration of transmission state for two types is shown in Figure 4.

Figure 4 illustrates the procedure of data transmission in 6LoWPAN using the acknowledged and unacknowlwdged transmission, respectively. The length of the IFS depends on the size of the frame that has been transmitted. The packet length greater than 18 bytes will be followed by a long IFS (LIFS is 40 symbols) and short frames by a short IFS (SIFS is 12 symbols). Thus, a mathematical analysis on transmission performance for the 6LoWPAN can be expressed in Acknowledged and Unacknowledged types.

\subsubsection{Goodput Analysis for Unacknowledged Transmission.} The goodput analysis model for unacknowledged transmission in both route-over and mesh-under routing schemes is presented in this section. We first obtain the expected transmission time for route-over and mesh-under routing schemes.

Consider

$$
\begin{gathered}
E_{\text {noack }}^{\mathrm{ro}}[\mathrm{tx}]=P_{\text {suc }} \cdot\left(T_{\text {data }}+T_{\mathrm{LIFS}}+\delta\right), \\
E_{\text {noack }}^{\mathrm{mu}}[\mathrm{tx}]=P_{\text {suc }} \cdot\left(T_{\text {data }}+T_{\mathrm{LIFS}}\right) .
\end{gathered}
$$

Equations (14) present the expected transmission time for route-over and mesh-under routing schemes, respectively. $T_{\text {data }}$ represents the transmission time for one IP fragment. $T_{\text {LIFS }}$ is LIFS period which is $640 \mu$ s. SIFS is used when the MPDU is smaller than or equal to 18 bytes. In addition, $\delta$ is the process delay for IP packet fragmentation and reassembly IP packet. We assume that the $\delta$ is around $2 \mathrm{~ms}$. 


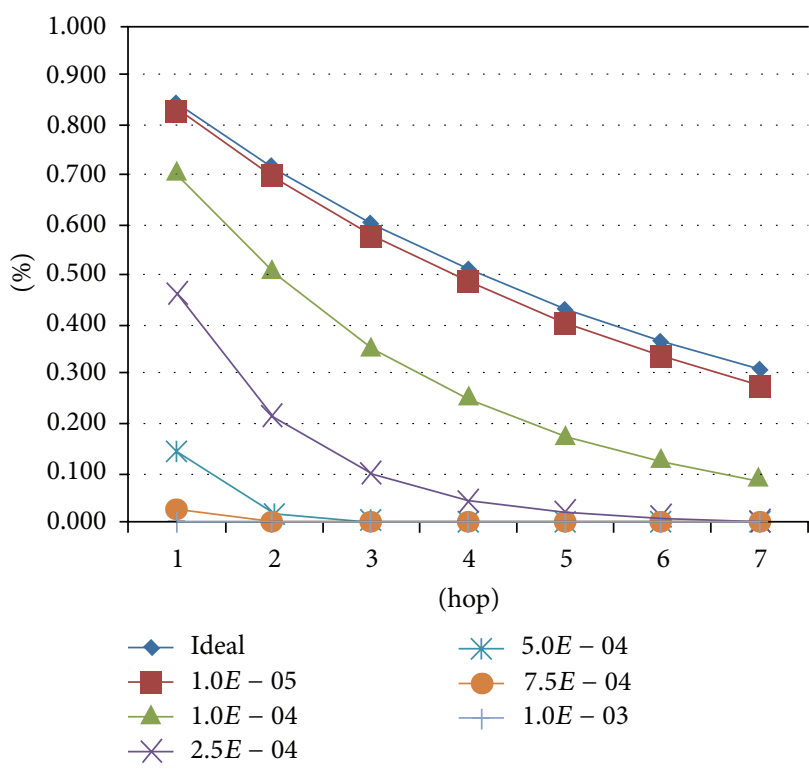

(a) Mesh-under

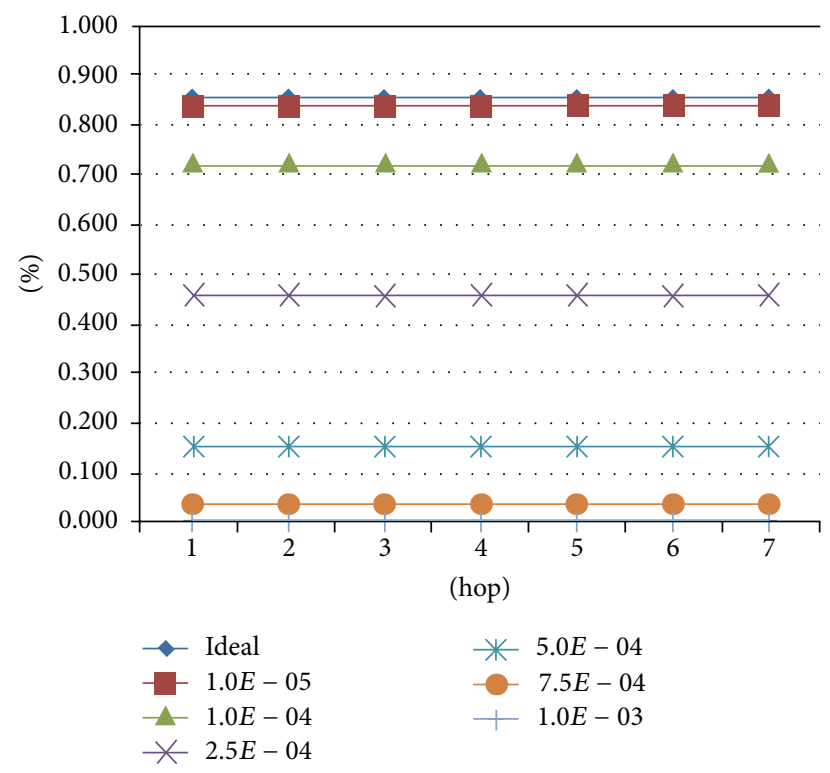

(b) Route-over

Figure 6: The successful IP packet transmission probability with 5 competing nodes for mesh-under and route-over routing schemes in 6LowPAN under error-prone channel condition.

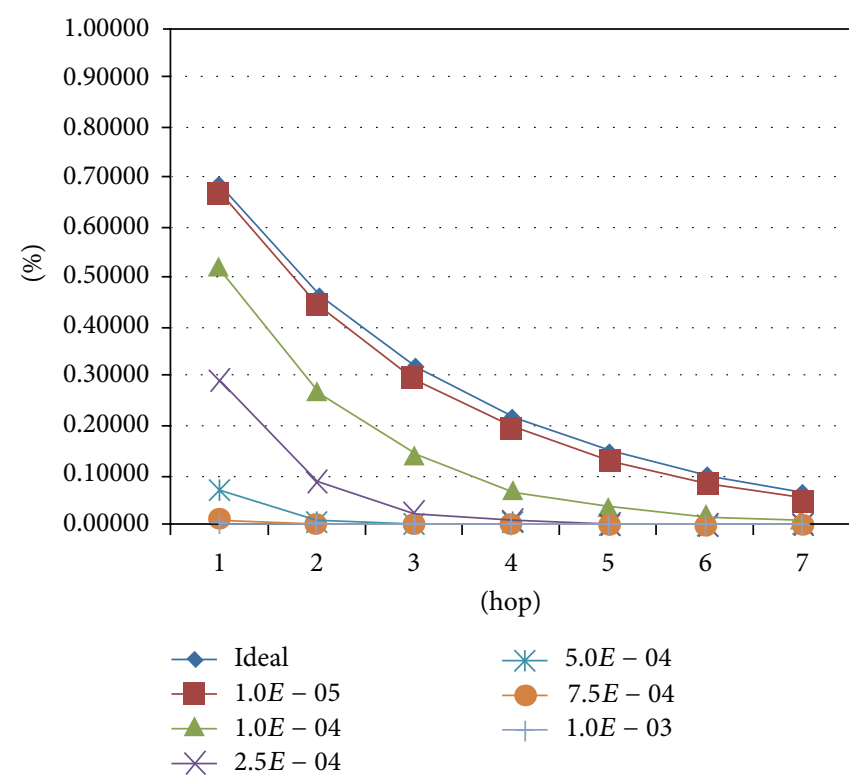

(a) Mesh-under

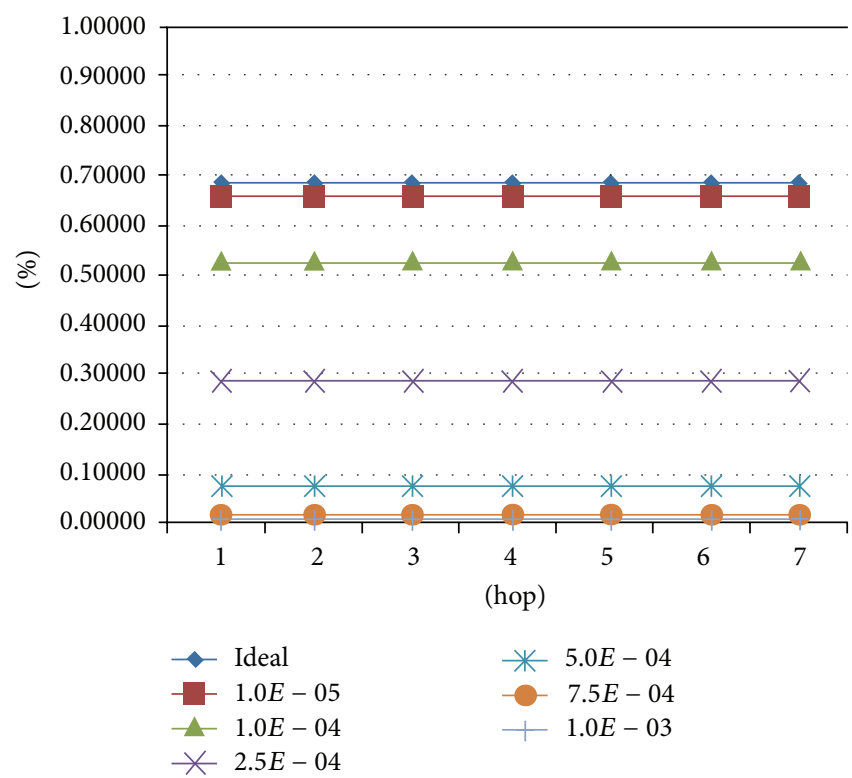

(b) Route-over

FIGURE 7: The successful IP packet transmission probability with 7 competing nodes for mesh-under and route-over routing schemes in 6LowPAN under error-prone channel condition.

Thus, the transmission goodput of route-over and meshunder routing schemes can be obtained from (15):

$$
\begin{aligned}
& S_{\text {noack }}^{\text {ro }}=\frac{P_{\text {suc }}^{\text {ro }} \cdot \mathrm{IP}_{\text {payload }}}{\left(E[\text { idle }]+E[\text { wait }]+E_{\text {noack }}^{\text {ro }}[\mathrm{tx}]\right) \cdot f \cdot h}, \\
& S_{\text {noack }}^{\text {mu }}=\frac{P_{\text {suc }}^{\mathrm{mu}} \cdot \mathrm{IP}_{\text {payload }}}{\left(E[\text { idle }]+E[\text { wait }]+E_{\text {noack }}^{\text {mu }}[\mathrm{tx}]\right) \cdot f \cdot h},
\end{aligned}
$$

where $P_{\text {suc }}^{\mathrm{ro}}$ and $P_{\mathrm{suc}}^{\mathrm{mu}}$ are the successful transmission probability for route-over and mesh-under routing schemes. $f$ and $h$ are the number of fragmentations and hop count from source to destination, respectively. $E$ [idle], $E$ [wait], and $E[\mathrm{tx}]$ represent the expected idle time, competing time, and transmission time, respectively.

5.3.2. Goodput Analysis for Acknowlwdged Transmission. In this section, we first obtain the expected transmission time 


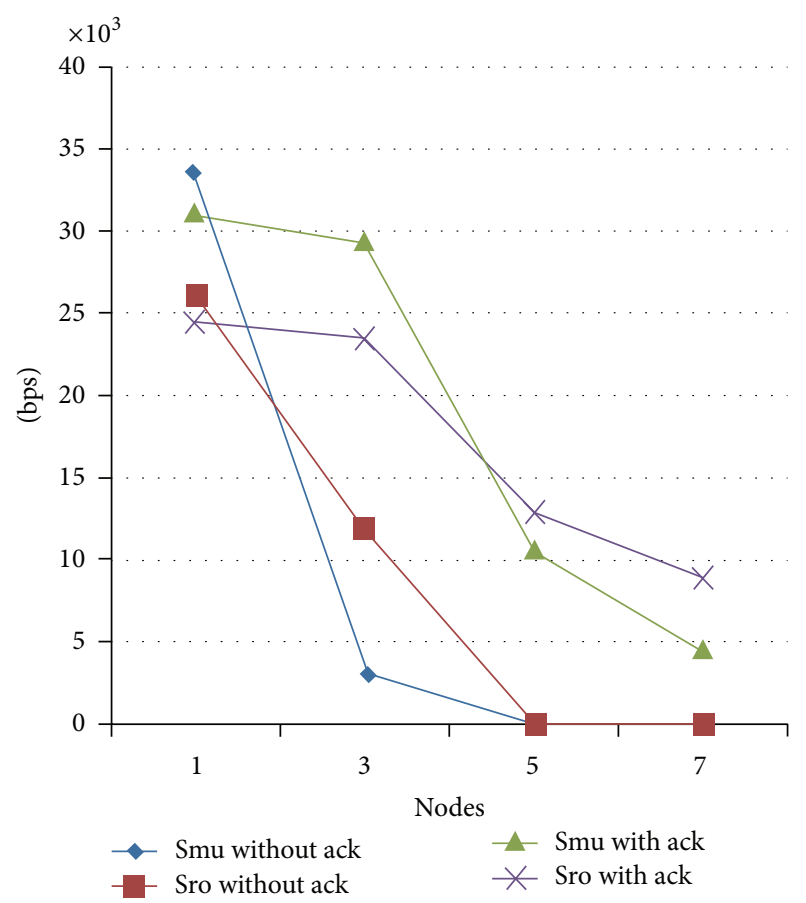

(a) $\mathrm{BER}=0$

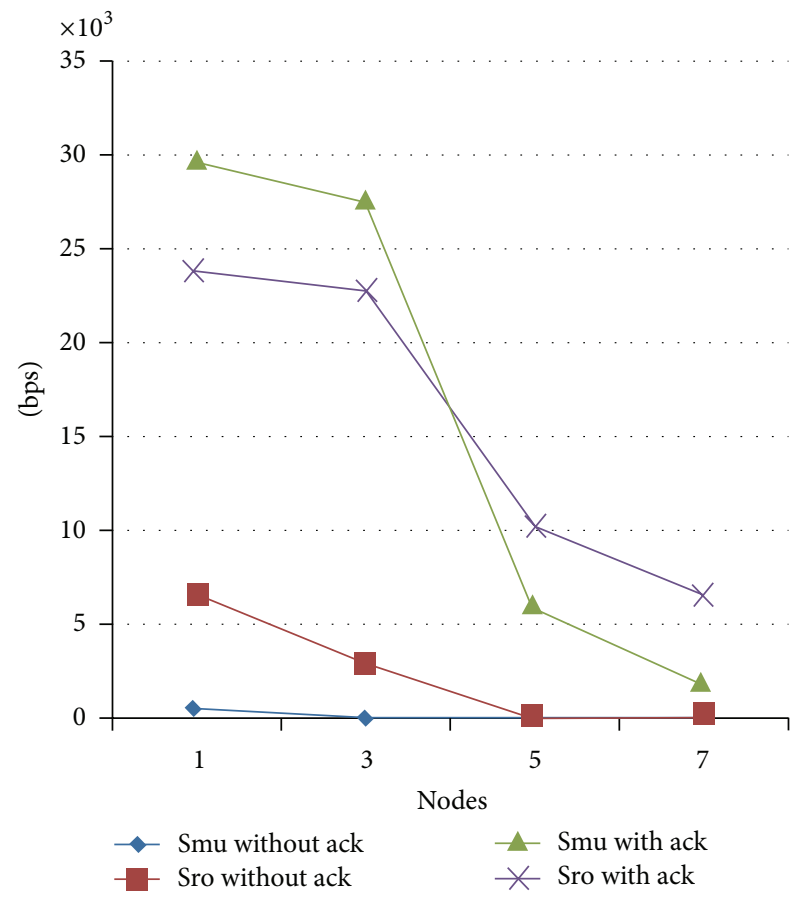

(c) $\mathrm{BER}=1 E-4$

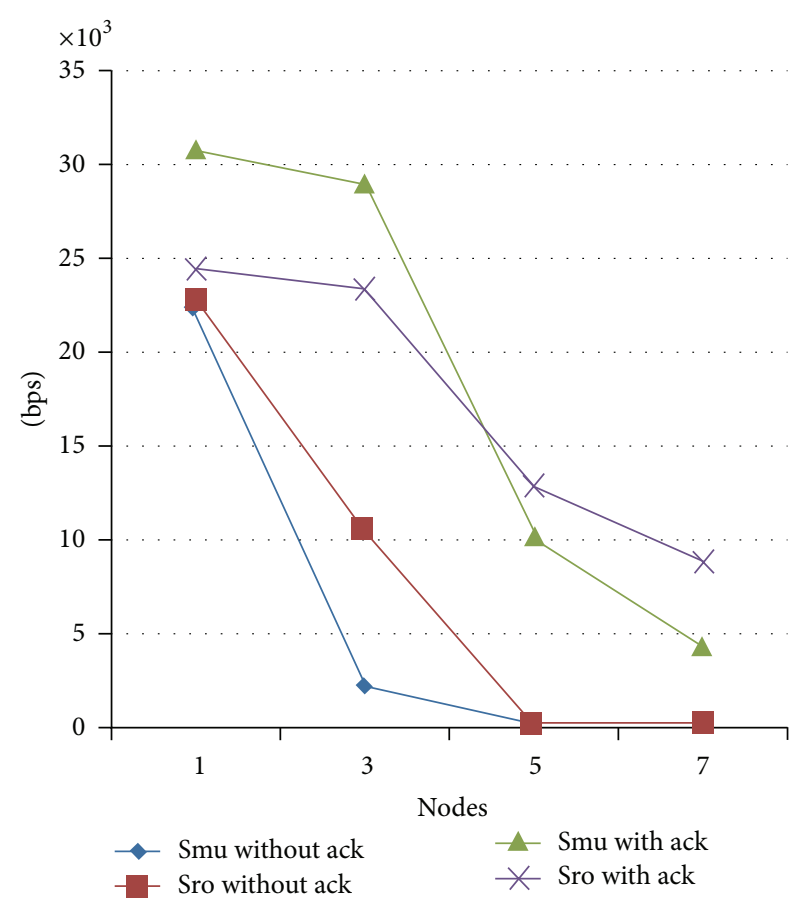

(b) $\mathrm{BER}=1 E-5$

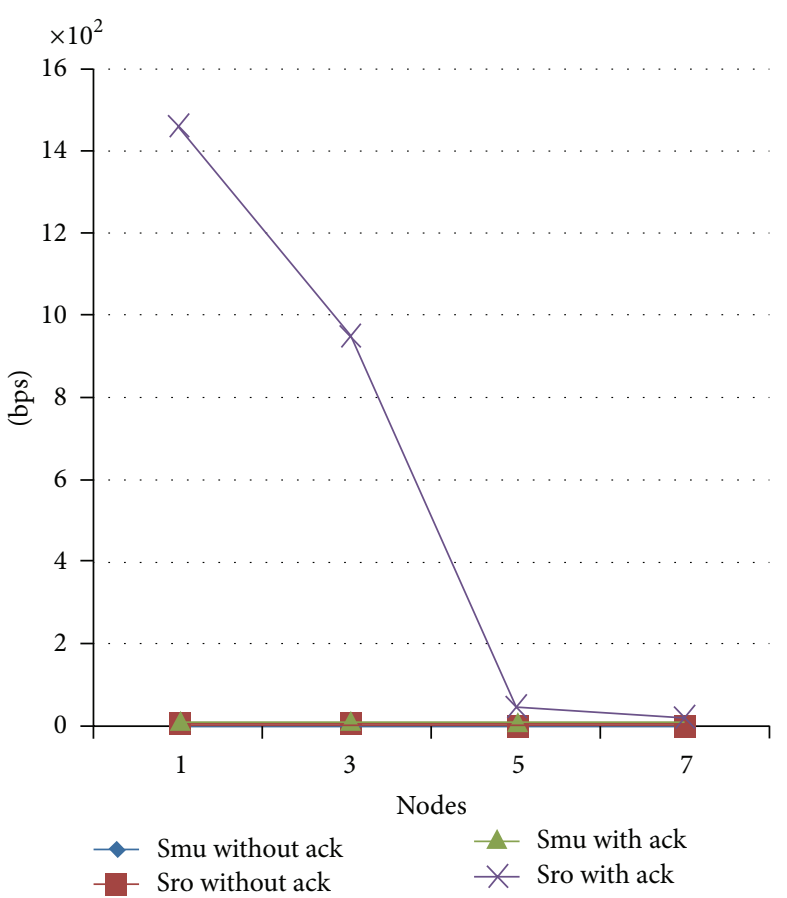

(d) $\mathrm{BER}=1 E-3$

FIGURE 8: Goodput for mesh-under and route-over routing schemes in 6LowPAN under error-prone channel condition.

for route-over and mesh-under routing schemes for acknowledged transmission:

$$
\begin{gathered}
E_{\mathrm{ack}}^{\mathrm{ro}}[\mathrm{tx}]=P_{\mathrm{suc}} \cdot\left(T_{\mathrm{data}}+T_{\text {turn around }}+T_{\mathrm{ack}}+T_{\mathrm{LIFS}}+\delta\right), \\
E_{\mathrm{ack}}^{\mathrm{mu}}[\mathrm{tx}]=P_{\mathrm{suc}} \cdot\left(T_{\mathrm{data}}+T_{\text {turn around }}+T_{\mathrm{ack}}+T_{\mathrm{LIFS}}\right) .
\end{gathered}
$$

$T_{\text {ack }}$ and $T_{\text {turn around }}$ are the acknowledgement transmission time $(352 \mu \mathrm{s})$, and turnaround time $(192 \mu \mathrm{s})$, respectively. If there is no acknowledgement, then turnaround time $T_{\text {turn around }}$ and $T_{\text {ack }}$ is equal to zero. Finally, the goodput of acknowledged transmission for route-over and mesh-under routing schemes in error-prone wireless environment can be 
TABLE 1: Numerical evaluation parameters.

IPv6 packet size

Number of fragments

Number of competing nodes

Hop counts

BER

computed as follows:

$$
\begin{aligned}
S_{\mathrm{ack}}^{\mathrm{ro}}= & P_{\text {suc }}^{\mathrm{ro}} \cdot \mathrm{IP}_{\text {payload }} \\
& \times\left(\left[\sum_{i=0}^{k}\left(1-P_{\mathrm{suc}}\right)^{i} \cdot(E[\text { idle }]+E[\text { wait }]\right.\right. \\
& \left.\left.\left.+E^{\mathrm{ro}}[\mathrm{tx}]\right)\right] \cdot f \cdot h\right)^{-1}, \\
S_{\mathrm{ack}}^{\mathrm{mu}}= & P_{\mathrm{suc}}^{\mathrm{mu}} \cdot \mathrm{IP}_{\text {payload }}, \\
& \times\left(\left[\sum_{i=0}^{k}\left(1-P_{\text {suc }}\right)^{i} \cdot(E[\mathrm{idle}]+E[\text { wait }]\right.\right. \\
& \left.\left.\left.+E^{\mathrm{mu}}[\mathrm{tx}]\right)\right] \cdot f \cdot h\right)^{-1},
\end{aligned}
$$

where macMaxFrameRetriesis 3, $f$, and $h$ are the number of fragmentations and hop count from source to destination, respectively.

\section{Performance Emulation Results for 6LoWPAN Routing Schemes in Error-Prone Channel Condition}

In this section, we present the numerical analysis results for 6LoWPAN routing schemes in error-prone channel condition. Our probabilistic model was emulated by PRISM [10]. Let the IPv6 packet size be minimum MTU (1280 bytes), and it will be fragmented into 14 maximum IEEE 802.15.4 frames (133 bytes), so called fragments. Assume $f$ fragments were sent from source to destination through $h$ hop counts. The evaluation parameters are shown in Table 1.

From results in figures 5, 6, and 7 , we observed that the number of competing nodes increases, the probability of successful transmission for two routing schemes are decreases due to the busy channel condition. The result shows that the rout-over routing is beneficial compared to the meshunder routing scheme since it reduces the probability of collisions from competing nodes and hop count. It is because that the transmission probability would resume to 1 due to that the route-over scheme reassembled all fragments for each hop. Therefore, the IP packet transmission successful probability would not decrease after multihops routing path. However, route-over consumes more delay time from hopby-hop fragments assembly and reassembly.
In Figure 8, as we can observe from both route-over and mesh-under routing schemes, the critical influence on the goodput in both schemes is the channel condition.

\section{Conclusions}

In this paper, we investigate the 6LoWPAN transmission performance by using the proposed mathematical model in 6LowPAN under varying number of competing nodes and error-prone channel condition. Analysis results show that route-over scheme has higher transmission probability than mesh-under.

\section{Acknowledgments}

This research was partially supported by the National Science Council of Republic of China, Taiwan under contracts, NSC102-2221-E-142-005 and NSC 101-2119-M-142-001 as well as National Taichung University regarding the MoE project (No. 1020035480A).

\section{References}

[1] Z. Shelby and C. Bormann, 6LoWPAN: The Wireless Embedded Internet, vol. 43 of Wiley Series on Communications Networking \& Distributed Systems, John Wiley \& Sons, New York, NY, USA, 2009.

[2] A. Ludovici, A. Calveras, and J. Casademont, "Forwarding techniques for IP fragmented packets in a real 6LoWPAN network," Sensors, vol. 11, no. 1, pp. 992-1008, 2011.

[3] G. Bianchi, "Performance analysis of the IEEE 802.11 distributed coordination function," IEEE Journal on Selected Areas in Communications, vol. 18, no. 3, pp. 535-547, 2000.

[4] S. Pollin, M. Ergen, S. C. Ergen et al., "Performance analysis of slotted carrier sense IEEE 802.15.4 medium access layer," IEEE Transactions on Wireless Communications, vol. 7, no. 9, pp. 33593371, 2008.

[5] Y. Zhang and F. Shu, "Packet size optimization for goodput and energy efficiency enhancement in slotted IEEE 802.15.4 networks," in Proceedings of the IEEE Wireless Communications and Networking Conference (WCNC '09), pp. 1-6, Budapest, Hungary, April 2009.

[6] F. Wang, D. Li, and Y. Zhao, "Analysis of CSMA/CA in IEEE 802.15.4," IET Communications, vol. 5, no. 15, pp. 2187-2195, 2011.

[7] C. Buratti and R. Verdone, "Performance analysis of IEEE 802.15.4 non beacon-enabled mode," IEEE Transactions on Vehicular Technology, vol. 58, no. 7, pp. 3480-3493, 2009.

[8] A. H. Chowdhury, M. Ikram, H. S. Cha et al., "Route-over vs mesh-under routing in 6LoWPAN," in Proceedings of the ACM International Wireless Communications and Mobile Computing Conference (IWCMC '09), pp. 1208-1212, June 2009.

[9] IEEE 802.15 Work Group, "Part 15.4: wireless medium access control (MAC) and physical layer (PHY) specifications for lowrate wireless personal area networks (LR-WPANs)," ANSI/IEEE Std 802.15.4, 2006.

[10] PRISM Website, http://www.prismmodelchecker.org/. 


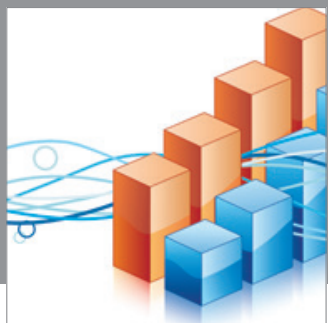

Advances in

Operations Research

mansans

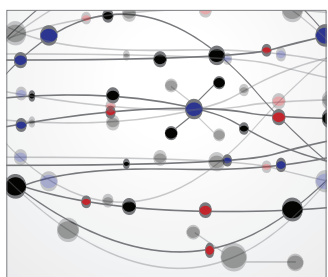

The Scientific World Journal
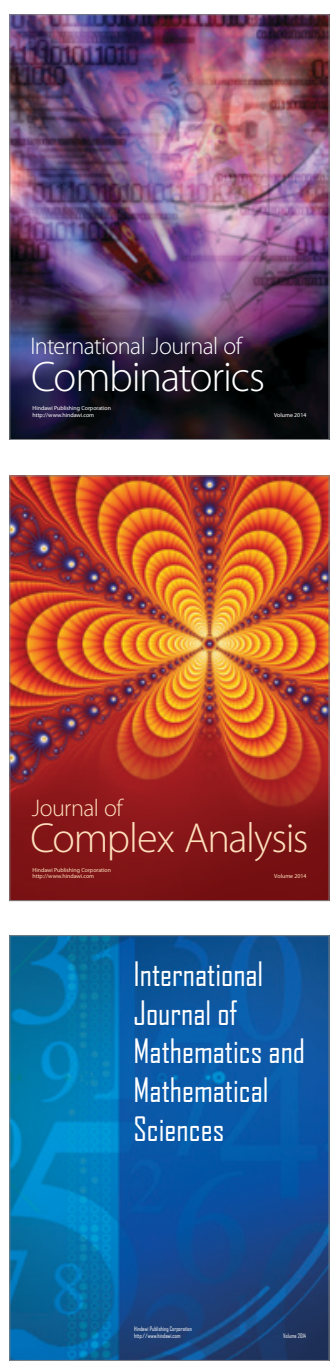
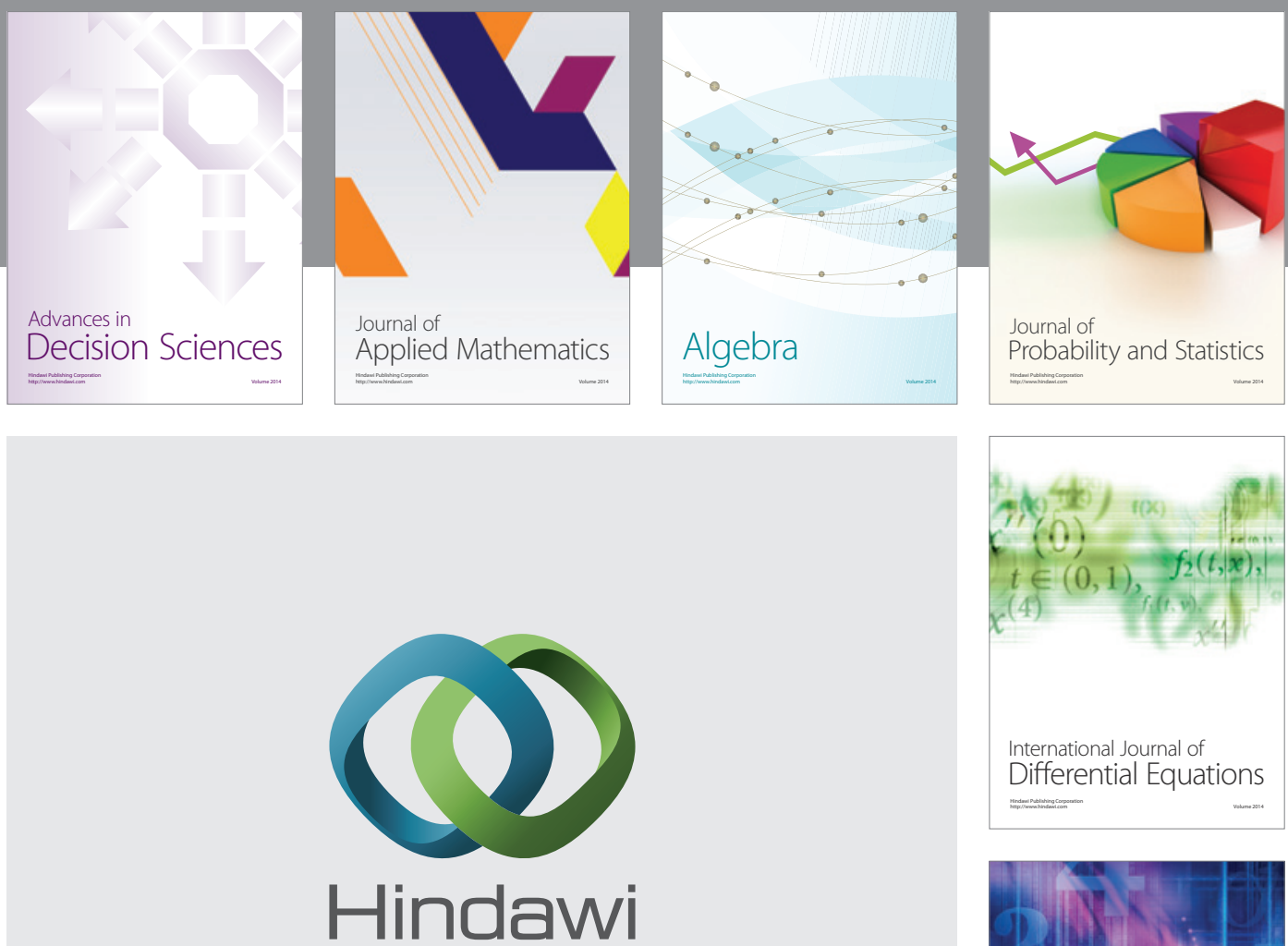

Submit your manuscripts at http://www.hindawi.com
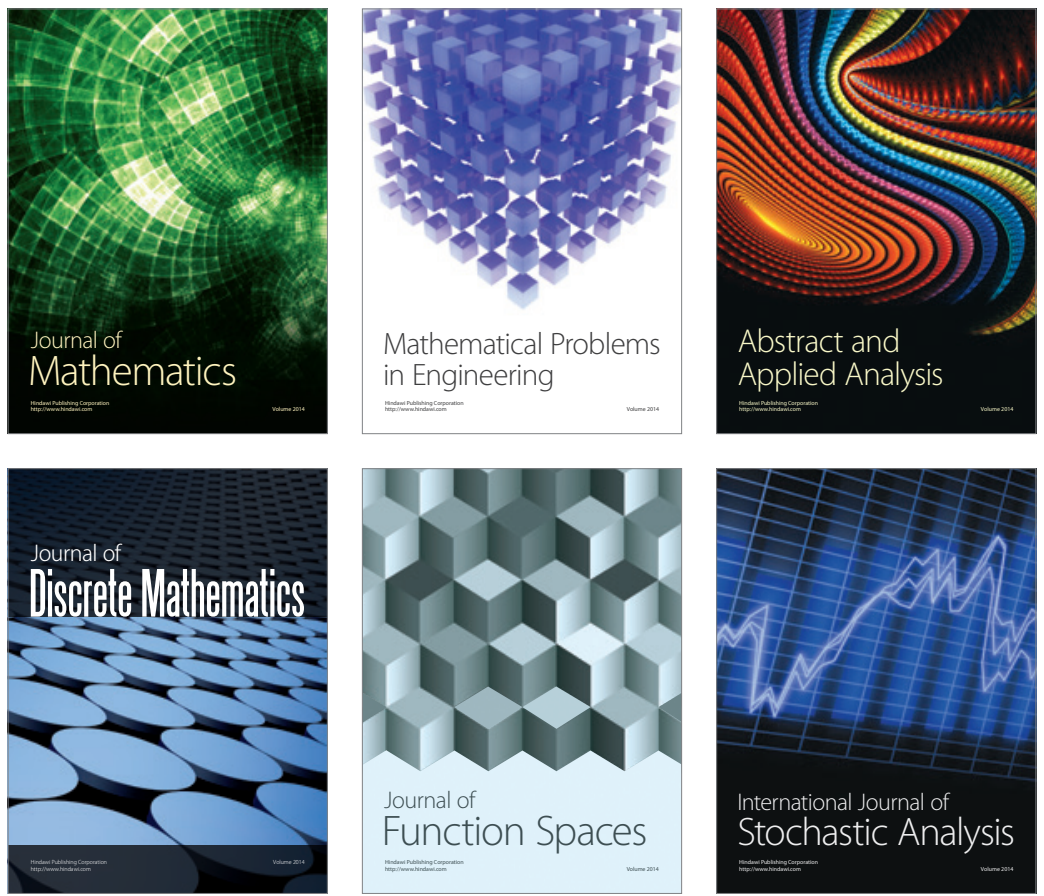

Journal of

Function Spaces

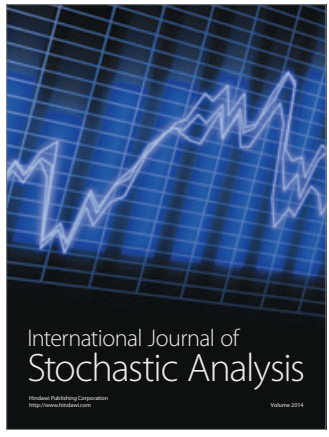

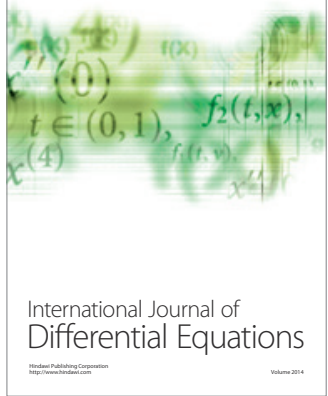
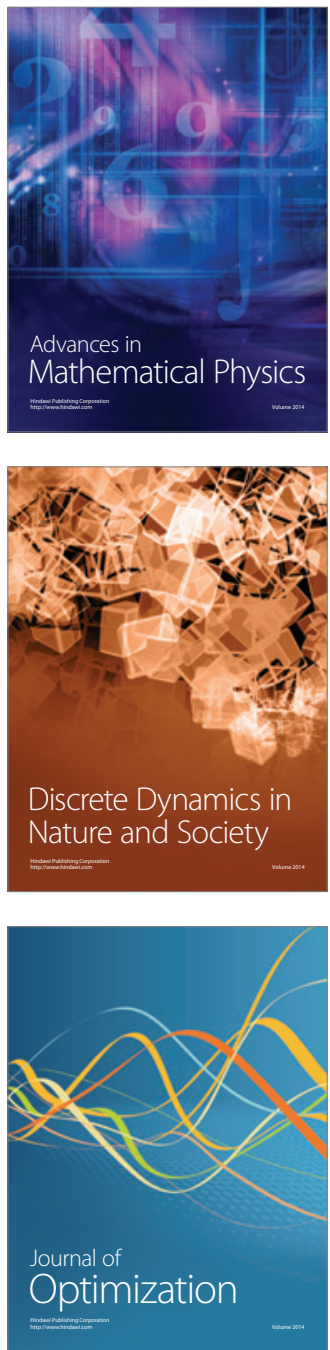